\title{
Fauna de flebotomíneos (Diptera: Psychodidae) em fragmentos florestais ao redor de conjuntos habitacionais na cidade de Manaus, Amazonas, Brasil. II. Estratificação horizontal.
}

\author{
Marlisson Augusto Costa FEITOSA ${ }^{1}$, Eloy Guillermo CASTELLÓN ${ }^{2}$
}

\section{RESUMO}

No período Janeiro a agosto de 1999, foi realizado um estudo sobre a presença de flebotomíneos em duas áreas de floresta residual, situadas próximas e no peri e domicilio de conjuntos habitacionais, na periferia da cidade de Manaus, Estado do Amazonas. O objetivo deste estudo foi verificar a fauna de flebotomíneos e observar possíveis mudanças de hábito principalmente nos vetores da leishmaniose cutânea. Foram realizadas coletas de flebotomíneos na bases de árvores no estrato entre 0 e 2 metros. As fêmeas foram dissecados para a procura de flagelados. Na Estrada do Turismo, capturamos 579 flebotomíneos de 12 espécies; 545 na floresta residual e 33 espécimes na área domiciliar. Na outra área, Cidade de Deus, foram capturados 976 exemplares de 21 espécies; 883 flebotomos na floresta residual e 72 espécimes no ambiente domiciliar. Todas as 375 fêmeas dissecadas foram negativas para flagelados. Lutzomyia umbratilis Ward \& Fraiha,1997 foi a espécie mais abundante em todos os ambientes de coletas.

PALAVRA-CHAVES

Diptera, Psychodidae, Flebotomíneos, Estratificação Horizontal.

\section{Sand fly faune (Diptera: Psychodidae) in forest fragments around bousing complexes in the Manaus municipality, Amazonas, Brazil. II. Horizontal stratification.}

\begin{abstract}
A study on phlebotomine sand fly occurrence was carried out from January to August, 1999, in two areas of residual forest (Estrada do Turismo and Cidade de Deus) located near lowcost housing complexes, in the periphery of Manaus (AM). The objetive of this study was to verify the sand fly fauna, and observe possible bebavior changes, mainly the cutaneous leishmaniasis vectors of CDC. Light traps were placed at a beight of one meter on the inside and outside of the dwellings; in addition, manual catches with glass tubes were made at the base of trees. Females were dissected in search of flagellates. In area 1 (Estrada do Turismo) 579 sand flies of 12 different species were captured, 545 in the residual forest and 33 specimens in the residential areas. In area 2 (Cidade de Deus) 976 specimens of 21 different species were captured, 883 sand flies were caught in the residual forest e 72 other specimens in the domiciliar areas. All three bundred and seventy five females dissected were negative for flagellate. Lutzomyia umbratilis Ward \& Fraiha, 1997 was the most abundant species in all collecting places.
\end{abstract}

\section{KEY WORDS}

Diptera, Psychodidae, Sand-fly, Horizon Stratification.

\footnotetext{
${ }^{1}$ Curso de Pós-graduação em Entomologia, PPG-BTRN do INPA/UFAM. e-mail - krill@inpa.gov.br

${ }^{2}$ INPA - CPCS Av. André Araújo no 2936, Petrópolis, 69083-000 Manaus - Amazonas, Brasil. e-mail - eloyc@inpa.gov.br
} 


\section{ACTA \\ AMAZONICA}

FAUNA DE FLEBOTOMINEOS (DIPTERA: PSYCHODIDAE) EM FRAGMENTOS

DE FLORESTA AO REDOR DE CONJUNTOS HABITACIONAIS NA CIDADE DE MANAUS,...

\section{INTRODUÇÃO}

As leishmanioses são consideradas pela Organização Mundial de Saúde (OMS) entre as seis doenças tropicais; depois da málaria, a segunda em importância, possuindo uma estimativa de aproximadamente 350 milhões de pessoas expostas e 15 milhões infectadas. Esta doença é considerada uma zoonose endêmica em mais de 82 países no mundo; a OMS estimou a ocorrência de aproximadamente 400 mil novos casos anualmente, sendo registrada no continente americano em quase todos os países, exceto Canadá, Chile e Uruguai (WHO,1996).

Segundo o Ministério da Saúde - Fundação Nacional de Saúde (MS-FNS, 1994) a Leishmaniose Tegumentar Americana no Brasil ocorre em praticamente todos os estados, principalmente na região Norte e Nordeste.

Barrett et al. (1991) concluíram que devido à incidência elevada, dificuldades na quimioterapia e capacidade de causar formas graves da doença, as leishmanioses cutâneas ou tegumentares na Amazônia representam um sério agravo à saúde dos habitantes de novos conjuntos residenciais, favelas e glebas de colonização agrícola em áreas de floresta primária, projetos de desenvolvimento e exploração de recursos naturais e para os integrantes das forças armadas em manobras na floresta.

Guerra et al. (1998) destacaram ainda, que ocorrência de casos de leishmaniose em Manaus alcançou o total de $56 \%$ (15.221 casos) do total de casos ocorridos no estado do Amazonas (25.917), no período de janeiro de 1985 a outubro de 1997, salientando ainda que ocorreu uma maior prevalência dessa enfermidade no período de novembro à maio, ou seja na época correspondente ao período chuvoso.

Mais recentemente, Andrade et al. (1999) estudaram a leishmaniose tegumentar no bairro Cidade de Deus, localizada na periferia de Manaus (AM), encontrando espécies vetoras dessa doença na floresta limítrofe com este bairro.

Rangel et al. (1999) relataram a infecção natural por $L$. (V) braziliensis Vianna, $1911 \mathrm{em}$ L. umbratilis no estado do Mato Grosso, sendo este flebótomo considerado o principal vetor de L. (V) guyanensis Floch, 1954 na Amazônia, relatando inclusive a alta atividade antropofilica desse inseto.

Arias \& Naiff (1981), em estudo realizado na área periurbana de Manaus (AM) em conjuntos habitacionais, constataram que o gambá Didelphis marsupialis, resiste bem à perturbação urbana, sendo inclusive atraído pelo lixo acumulado nos arredores das casas. $D$. marsupialis foi encontrado naturalmente infectado por Leishmania (V) guyanensis.

Em trabalho realizado em mancha de floresta próxima ao conjunto habitacional Parque das Laranjeiras, na cidade de Manaus, Arias \& Freitas (1982) coletaram 6.955 espécimes de flebotomíneos, sendo que deste total 6.560 eram Lutzomyia umbratilis.

Barrett et al. (1991) afirmaram que cerca de 5\% das fêmeas de Lutzomyia umbratilis capturadas em armadilhas de luz tipo CDC, estavam infectadas com Leishmania guyanensis. Segundo Barrett et al. (1991) tem-se coletado Lutzomyia umbratilis entre as casas até 80 metros da margem da floresta, e a ocorrência de leishmaniose em crianças de colo e deficientes físicos sugere que alguma transmissão ao homem esteja ocorrendo no ambiente domiciliar. No entanto, Leishmania guyanensis é peridomiciliar apenas no restrito sentido de as casas são construídas próximos do ambiente natural do transmissor.

Paes (1991) em um inquérito entomológico realizado no bairro Cidade Nova em Manaus - AM, encontrou que Lutzomyia umbratilis comportou $56,2 \%$ da fauna de flebotomíneos, sendo que 5.865 espécimes foram coletados na mancha de floresta próximas às residências, 17 espécimes no peridomicílio e 7 espécimes no próprio domicilio. Flebotomíneos infectados com Leishmania foram capturados apenas em mancha de floresta, onde constituíram 3,7\% do total coletado neste ambiente.

Segundo Biancardi (1991) a realização de estudos sobre epidemiologia e ecologia das leishmanioses, e incriminação de vetores e seus hábitos, são fundamentais para a aplicação de medidas profiláticas. Portanto neste trabalho verificaremos a infecção natural em flebotomíneos e a ocorrência de possíveis mudanças no comportamento de Lutzomyia umbratilis, encontrados nas manchas de floresta residual na periferia de Manaus.

O objetivo deste estudo foi verificar a fauna natural de flebotomíneos em áreas residenciais e orla de fragmentos de floresta ao redor de conjuntos habitacionais de Manaus.

\section{MATERIAL E MÉTODOS}

As capturas dos flebotomíneos, utilizando armadilhas luminosas tipo CDC "miniatura" (Hausher's Machile Works ${ }^{\circledR}$, New Jersey, EUA) foram realizadas durante 4 dias de uma semana a cada mês, nos horários de 18:00 às 06:00 horas de janeiro a agosto de 1999, simultaneamente nas duas áreas.

Para verificar a estratificação horizontal, da fauna de flebotomíneos existente nesses conjuntos habitacionais, foram utilizadas 4 armadilhas luminosas tipo CDC, colocadas no interior e exterior dos domicílios (mínimo de 2 casas por rua) em ruas que situavam a aproximadamente 150 a 200 metros de mancha de floresta. Nesta modalidade de coleta as armadilhas eram colocadas apenas a um metro de altura, e preferencialmente colocadas em quartos eram rotativos os locais de coleta, ou seja, eram deslocadas de seu local a cada dois dias e montadas noutros locais e retiradas no horário acima citado.

As coletas dos flebotomíneos nas bases de árvores, foram realizados através de captura manual com tubos de vidro; utilizamos os seguintes critérios: foi delimitada uma área de aproximadamente 1 hectare na floresta residual, e esta dividida em 4 partes, coletando-se durante uma hora, aproximadamente, em cada parte; nestas partes foram selecionadas de oito a onze árvores para estas coletas. Tais coletas ocorreram em 4 dias de uma semana a cada mês, sendo realizadas em dois locais de uma das duas áreas cada dia. 


\section{ACTA \\ AMAZONICA}

FAUNA DE FLEBOTOMÍNEOS (DIPTERA: PSYCHODIDAE) EM FRAGMENTOS

DE FLORESTA AO REDOR DE CONJUNTOS HABITACIONAIS NA CIDADE DE MANAUS,...
As coletas de flebotomíneos em tronco das árvores eram realizadas no período da manhã entre 08:00 e 10:00 h, no estrato de 0 a 2 metros de altura, durante aproximadamente 10 minutos para cada árvore. Foram escolhidas as árvores com mais de 0,5 metro de circunferência com presença de raízes tabulares (sapopema), tipo de casca (lisa ou rugosa) presença ou não de cupinzeiros e cobertura do dossel ampla.

Para verificação da domiciliação de $L$. umbratilis, foi realizada a procura ativa das espécies durante o dia, no interior das casas, escolhendo-se as casas onde na noite anterior tinham sido coletados flebótomos, com armadilhas CDC. Para isso, no período da manhã, utilizamos uma lanterna e capturador manual, onde observando a presença de flebótomos nas paredes das residências e moradias de animais.

Todo material dessas coletas, foi acondicionado em caixas de poliestireno com umidade interna mantida através de algodão umedecido, e levado para o laboratório de Entomologia do INPA, onde eram dissecados os espécimes vivos de flebotomíneos montados em lâminas e identificados, de acordo com critérios apresentados adiante.

A dissecação das fêmeas de flebótomos foi realizada para observação de flagelados presentes no intestino dos insetos. A técnica utilizada para dissecação, foi a de Ryan et al. (1987), onde as fêmeas coletadas eram dissecadas em microscópio estereoscópio, com auxílio de estiletes flambados, sobre lâminas esterilizadas em álcool por 2 horas e colocadas em estufa a $150^{\circ} \mathrm{C}$ por 12 horas, contendo duas gotas separadas de soro fisiológico $(0,5 \%$ ou $0,9 \%)$.

As fêmeas foram identificadas pelas características de suas espermatecas e os manchos de todas as espécies coletadas, foram montados em lâminas e devidamente identificados segundo as chaves de Young \& Duncan (1994).

\section{RESULTADOS}

Durante os oito meses em que foi realizado o trabalho de campo, capturamos 1.555 flebotomíneos, distribuídos em 23 espécies.

Na área 1 capturamos 579 exemplares de flebotomíneos distribuídos em 12 espécies; 545 espécimes foram coletados na floresta residual e 33 no ambiente domiciliar. Na Área 2 foram capturados um total 976 exemplares, pertencentes a 21 espécies, 883 flebótomos foram coletados na floresta residual e 72 espécimes no ambiente domiciliar.

\section{Coletas no ambiente domiciliar na Estrada do Turismo (Área 1)}

No ambiente domiciliar foram coletados 34 exemplares de flebotomíneos, 22 no intradomicílio e 12 outros espécimes no peridomicílio. Estes flebotomíneos coletados neste ambiente encontram-se agrupados em 15 espécies (Tabela 1). No intradomicílio, de acordo com o sexo, as fêmeas foram mais abundantes do que os machos, 63,6\% e $36,4 \%$ respectivamente. $L$. umbratilis se destacou como a espécie mais abundante apresentando 5 indivíduos durante o período de coleta, seguido por L. trichopyga com 3 exemplares e L. ubiquitalis, L. eurypyga e L. rorotaensis representados com 2 indivíduos cada uma; foram também registrados nesse ambiente, as espécies $L$. amazonensis, $L$. anduzei, L. davisi, L. furcata, L. inflata, L. shannoni e $L$. spatbotrichia com um indivíduo cada (Tabela 1).

No peridomicílio, segundo o sexo houve equilíbrio numérico entre fêmeas e machos ( $50 \%$ cada). Dentre as espécies coletadas, L. umbratilis e L. ubiquitalis também destacaram-se como as mais abundante apresentando 5 indivíduos cada uma, também L. infraspinosa e L. dreisbachi foram registrados neste ambiente com um indivíduo de cada espécie; L. dreisbachi (grupo Dreisbachi) foi coletado exclusivamente neste ambiente e nesta área (Estrada do Turismo), durante todo o tempo de nosso estudo (Tabela 1).

\section{Coletas em bases de árvores}

Em nosso trabalho na área 1, colecionamos neste ambiente, 545 flebotomíneos, distribuídos em 9 espécies e quatro subgêneros (Nyssomyia, Psatbyromyia, Trichophoromyia e Viannamyia) e grupo(Oswaldoi). Verificada a proporção quanto ao sexo, os machos $(54,68 \%)$ foram mais numerosos que as fêmeas $(45,32 \%)$ (Tabela 1$)$.

Neste ambiente de coleta a espécie mais abundante foi L. umbratilis, tendo sido capturados 523 indivíduos (288 machos e 235 fêmeas), o que representou 95,96\% do total de flebotomíneos deste ambiente. Do mesmo subgênero foi também coletado $L$. flaviscutellata com 2 indivíduos (1 macho e 1 fêmea). As espécies do subgênero Psatbyromyia foram as mais representadas, depois do subgênero Nyssomyia, com 3 espécies diferentes registradas nesta área (L. dendrophyla, L. lutziana e L. shannoni), apresentando 13 flebótomos (Tabela 1).

\section{Dissecação dos flebotomíneos da Estrada do Turismo}

De todos os flebotomíneos coletados nesta área, foi dada preferência para dissecção às fêmeas grávidas ou ingurgitadas coletadas das armadilhas localizadas no intra e peridomicílio e das coletados em bases de árvores, por terem maior possibilidade de apresentarem infecção natural por leishmanias. Destacamos que das 156 fêmeas capturadas, não foi conseguido nenhum exemplar com infecção por leishmanias.

\section{Coletas no ambiente domiciliar na área 2 (Cidade de Deus)}

Neste ambiente, coletamos 72 flebótomos, distribuídos em 16 espécies, das quais nove (9) foram encontradas no intradomicílio e 11 no peridomicílio, deste total, 35 espécimes foram coletados no intradomicílio e 37 outros no peridomicílio. 
Tabela 1 - Flebotomíneos coletados na Área 1 (Estrada do Turismo) Município de Manaus, Amazonas, Brasil e discriminados de acordo com a espécie, sexo (Mmacho; F- fêmea) e ambiente de coleta (intra e peridomicílio, e bases das árvores), no período de Janeiro a Agosto de 1999.

\begin{tabular}{|c|c|c|c|c|c|c|c|}
\hline \multirow[t]{2}{*}{ Espécie } & \multicolumn{2}{|c|}{$\begin{array}{c}\text { Intra } \\
\text { domicílio }\end{array}$} & \multicolumn{2}{|c|}{$\begin{array}{c}\text { Peri } \\
\text { Domicílio }\end{array}$} & \multicolumn{2}{|c|}{$\begin{array}{l}\text { Base de } \\
\text { árvores }\end{array}$} & \multirow[t]{2}{*}{ Total } \\
\hline & $M$ & $F$ & $M$ & $F$ & $M$ & $F$ & \\
\hline L. amazonensis & 0 & 1 & 0 & 0 & 0 & 0 & 1 \\
\hline L. anduzei & 0 & 1 & 0 & 0 & 0 & 0 & 1 \\
\hline L. davisi & 0 & 1 & 0 & 0 & 0 & 0 & 1 \\
\hline L. dendrophyla & 0 & 0 & 0 & 0 & 2 & 3 & 5 \\
\hline L. dreisbachi & 0 & 0 & 0 & 1 & 0 & 0 & 1 \\
\hline L. eurypyga & 1 & 1 & 0 & 0 & 1 & 0 & 3 \\
\hline L. flaviscutellata & 0 & 0 & 0 & 0 & 1 & 1 & 2 \\
\hline L. furcata & 0 & 1 & 0 & 0 & 0 & 0 & 1 \\
\hline L. inflata & 1 & 0 & 0 & 0 & 0 & 0 & 1 \\
\hline L. infraspinosa & 0 & 0 & 0 & 1 & 0 & 0 & 1 \\
\hline L. lutziana & 0 & 0 & 0 & 0 & 1 & 0 & 1 \\
\hline$L$ rorotaensis & 0 & 2 & 0 & 0 & 2 & 2 & 6 \\
\hline L. ruii & 0 & 1 & 0 & 0 & 0 & 0 & 1 \\
\hline L. shannoni & 1 & 0 & 0 & 0 & 3 & 4 & 8 \\
\hline L. spathotrichia & 0 & 1 & 0 & 0 & 0 & 0 & 1 \\
\hline L. trichopyga & 2 & 1 & 0 & 0 & 0 & 0 & 3 \\
\hline L. tuberculata & 0 & 0 & 0 & 0 & 0 & 1 & 1 \\
\hline L. ubiquitalis & 1 & 1 & 4 & 1 & 0 & 1 & 8 \\
\hline L. umbratilis & 2 & 3 & 2 & 3 & 288 & 235 & 533 \\
\hline Sub -Total & 8 & 14 & 6 & 6 & 298 & 247 & 579 \\
\hline Total & \multicolumn{2}{|c|}{22} & \multicolumn{2}{|c|}{12} & \multicolumn{2}{|c|}{545} & 579 \\
\hline
\end{tabular}

\section{Flebotomíneos encontrados na Base de árvores}

Durante a realização deste estudo, na área 2 (Cidade de Deus), colecionamos um total de 883 flebotomíneos, distribuídos em 13 espécies. Sendo que na relação entre os sexos, os machos com 534 indivíduos (60,36\%) foram bem mais abundantes do que as fêmeas com 349 indivíduos (39,64\%).

Entre os flebotomíneos coletados neste ambiente L. umbratilis (subgênero Nyssomyia) destacou-se como a espécie mais abundante, com 818 indivíduos capturados, o que representou 92,64\% do total de flebotomíneos neste ambiente. Pertencente a este subgênero também foram registradas nestes locais as espécies $L$. olmeca nociva, L. flaviscutellata e $L$. anduzei $\operatorname{com} 6,4 \mathrm{e}$ 1 indivíduos respectivamente. Outras espécies representadas nesta área foram, L. shannoni 15 indivíduos, $L$. dendrophyla 14 indivíduos $L$. scaffi com 3 indivíduos, e L. rorotaensis (grupo Oswaldoi) com 16 flebótomos. Neste local foram também registradas as espécies, L. amazonensis, L. davisi (subgênero Psychodopygus) com três flebótomos coletados, L. (Trichophoromyia) ruii e $L$. (Trichopygomyia) trichopyga com um exemplar cada uma (Tabela 2).

Entre os flebotomíneos coletados nesta área, foram preferencialmente dissecados as fêmeas grávidas ou as que estivessem ingurgitadas. Não foi conseguido nenhum com infecção por leishmanias.
Foi observado no interior das moradias que a proporção de fêmeas com $62,86 \%$ foi superior à de machos com $37,14 \%$. A espécie L. umbratilis destacou-se como a mais abundante entre as espécies coletadas, representado por 18 indivíduos porém apresentou o maior número de machos (10). Outras espécies registradas foram $L$. rorotaensis com 5 flebótomos, L. trichopyga com 3 exemplares e L. davisi, L. ruii e L. sericea com 2 indivíduos cada. Foi coletado ainda nesse ambiente, um exemplar das espécies $L$. nematoducta, L. paraensis e $L$. spatbotrichia (Tabela 2). Na área que compunha o peridomicílio houve uma diferença pouco significativa entre o número de fêmeas com 18 indivíduos (48,65\%) e de machos com 19 indivíduos (51,35\%).

Dentre as espécies coletadas, L. umbratilis e $L$. rorotaensis, destacaram-se como as mais abundantes apresentando 10 e 7 espécimes respectivamente, seguidos por L. anduzei e L. trichopyga com 5 indivíduos cada uma; L. eurypyga, L. infraspinosa e shannoni estiveram presentes neste ambiente de coleta com 2 flebótomos cada uma e $L$. antunesi, L. brachipyga, L. davisi e L. flaviscutellata foram também registrados no peridomicílio representados por um indivíduo cada espécie (Tabela 2).

\section{DISCUSSÃO}

\section{Coletas em ambiente Domiciliar}

Segundo Marzochi \& Marzochi (1997) dois diferentes padrões epidemiológicos de transmissão de LTA ocorrem na região Amazônica: silvestre-florestal - onde a doença é uma zoonose de animais silvestres, com ocorrência de alguns casos humanos em áreas de colonização recente devido a penetração do homem em ambiente silvestre; e silvestre-periflorestal - onde a doença é uma antropozoonose, que ocorre em áreas de ocupação situadas dentro do raio de vôo dos vetores silvestres.

Cada espécie de Leishmania apresenta um quadro ecológico associado, com hospedeiros reservatórios, vetor e distribuição geográfica próprias. Esses diferentes tipos de associações podem resultar num ciclo de transmissão específico, onde somente alguns flebotomíneos são importantes vetores.

O número de 13 espécies coletadas na área 1 e 16, capturadas na área $2 \mathrm{em}$ ambiente domiciliar (intra e peridomicílio), foi semelhante ao encontrado por Paes 


\section{ACTA AMAZONICA}

(1991) que capturou 13 espécies em seu estudo na Cidade Nova, bairro periférico da cidade de Manaus-AM. Deste total, 4 das espécies capturadas na área 1 e 5 espécies na área 2, também foram capturadas neste estudo, entre elas $L$. umbratilis e L. anduzei incriminados como principais vetores de leishmaniose tergumentar no Estado.

Nossos resultados de coletas no intra e periomicílio mostraram que aproximadamente $40 \%$ das espécies (L. umbratilis, L. ubiquitalis, L. rorotaensis, L. anduzei $\mathrm{e}$ L. davisi) foram coletadas na área 1 e 30\% das espécies (L. umbratilis, L. anduzei, L. davisi, L. rorotaensis e L. trichopyga) capturadas no intra e peridomicílio na área 2; embora com número reduzido de indivíduos, teriam capacidade de adaptar-se a ambientes sobre ação antropica, devido serem capturadas em quantidades relevantes na floresta residual e serem detectadas em todos os ambientes de coleta deste estudo. Essas mudanças de hábitos dos flebotomíneos, como conseqüência da expansão urbana desordenada, foram constatadas em varias cidades do sudeste e sul brasileiro por Gomes et al. (1982) e Teodoro

Tabela 2 - Flebotomíneos coletadas na Área 2 (Cidade de Deus) Município de Manaus, Amazonas, Brasil e discriminados de acordo com a espécie, sexo (Mmacho; F- fêmea) e local de coleta (intra e peridomicílio, e bases das árvores), no período de Janeiro a Agosto de 1999.

\begin{tabular}{|c|c|c|c|c|c|c|c|}
\hline \multirow[t]{2}{*}{ Espécie } & \multicolumn{2}{|c|}{$\begin{array}{c}\text { Intra } \\
\text { domicílio }\end{array}$} & \multicolumn{2}{|c|}{$\begin{array}{c}\text { Peri } \\
\text { Domicílio }\end{array}$} & \multicolumn{2}{|c|}{$\begin{array}{l}\text { Base de } \\
\text { árvores }\end{array}$} & \multirow[t]{2}{*}{ Total } \\
\hline & $M$ & $F$ & $M$ & $F$ & $M$ & $F$ & \\
\hline L. amazonensis & 0 & 0 & 0 & 0 & 0 & 1 & 1 \\
\hline L. anduzei & 0 & 0 & 2 & 3 & 1 & 0 & 6 \\
\hline L. antunesi & 0 & 0 & 0 & 1 & 0 & 0 & 1 \\
\hline L. brachypyga & 0 & 0 & 1 & 0 & 0 & 0 & 1 \\
\hline L. davisi & 0 & 2 & 1 & 0 & 0 & 2 & 5 \\
\hline L. dendrophyla & 0 & 0 & 0 & 0 & 11 & 3 & 14 \\
\hline L. eurypyga & 0 & 0 & 2 & 0 & 0 & 0 & 2 \\
\hline L. flaviscutellata & 0 & 0 & 0 & 1 & 4 & 0 & 5 \\
\hline L. infraspinosa & 0 & 0 & 0 & 2 & 0 & 0 & 2 \\
\hline L. nematoducta & 1 & 0 & 0 & 0 & 0 & 0 & 1 \\
\hline L. olmeca nociva & 0 & 0 & 0 & 0 & 6 & 0 & 6 \\
\hline L. paraensis & 0 & 1 & 0 & 0 & 0 & 0 & 1 \\
\hline$L$ rorotaensis & 1 & 4 & 1 & 6 & 12 & 4 & 28 \\
\hline L. ruii & 0 & 2 & 0 & 0 & 0 & 1 & 3 \\
\hline L. scaffi & 0 & 0 & 0 & 0 & 3 & 0 & 3 \\
\hline L. sericea & 0 & 2 & 0 & 0 & 0 & 0 & 2 \\
\hline L. shannoni & 0 & 0 & 0 & 2 & 11 & 4 & 17 \\
\hline L. spathotrichia & 0 & 1 & 0 & 0 & 0 & 0 & 1 \\
\hline L. trichopyga & 1 & 2 & 5 & 0 & 0 & 1 & 9 \\
\hline L. tuberculata & 0 & 0 & 0 & 0 & 0 & 1 & 1 \\
\hline L. umbratilis & 10 & 8 & 7 & 3 & 486 & 332 & 846 \\
\hline Sub -Total & 13 & 22 & 19 & 18 & 534 & 349 & 955 \\
\hline Total & \multicolumn{2}{|c|}{35} & \multicolumn{2}{|c|}{37} & \multicolumn{2}{|c|}{883} & 955 \\
\hline
\end{tabular}

et al. (1993), também devido a alterações de floresta, que provocaram a restrição de espaços ecológicos para os flebotomíneos (Tesh, 1995).

Dentre as espécies coletadas neste ambiente em nosso estudo, 5 são vetoras ou suspeitas de transmitirem a leishmaniose (L. umbratilis, L. anduzei, L. flaviscutellata, L. furcata e L. ubiquitalis), possuindo hábitos antropofilicos; destes flebótomos destacam-se os dois primeiros que são vetores de L. (V.) guyanensis, responsáveis pela maioria dos casos de leishmaniose tergumentar na Amazônia. Lainson (1983) e Arias et al. (1987) citaram que L. flaviscutellata estava envolvida na transmissão de $L$. amazonensis e recentemente Lainson et al.(1992) encontraram a L. ubiquitalis infectada com L. lainsoni que causa leishmaniose tegumentar ocasionalmente no homem. Estes autores consideraram ainda que em certas condições, este inseto demonstra hábitos antropofilicos. A captura destas espécies nesse ambiente, pode contribuir para a ocorrência de transmissão periurbana de leishmaniose tegumentar em Manaus.

Andrade et al. (1999) frisaram que a transmissão de leishmaniose tegumentar na cidade de Manaus seria do tipo periflorestal, em razão do reduzido número de indivíduos e espécies (L. umbratilis, L. anduzei e $L$. flaviscutellata) coletados em dois conjuntos desta cidade durante um período de três meses. Barrett \& Senra (1986) apresentaram a mesma conclusão, porém destacaram a presença de flebotomíneos (L. umbratilis) até 80 metros da margem da floresta, o qual seria um indicativo de adaptação e raio de dispersão amplo desses dípteros, indicados por Lainson (1983) como de caráter estritamente silvestre. Nesse estudo o número espécies e a presença de vários vetores da LTA, intradomicilio e peridomicilio nessas áreas reforçam o argumento de adaptação e abrem a possibilidade de possível substituição de espécies de flebotomíneos na transmissão de LTA na periferia da Cidade de Manaus.

\section{Coletas em bases de árvores}

Encontramos em nossas coletas em troncos de árvores 16 espécies de flebotomíneos (9 na área 1 e 13 na área 2) com 6 destas comuns nas duas áreas. Estas espécies pertencem a 6 subgênero, onde Nyssomyia se destaca pelo maior número de espécies (4) e indivíduos coletados (>90\% nas duas áreas), com a espécies L. umbratilis mais abundante nesse ambiente e com aproximadamente $95 \%$ e $93 \%$ do total de flebotomíneos coletados na área 1 e 2 respectivamente; o segundo subgênero mais 


\section{ACTA \\ AMAZONICA}

FAUNA DE FLEBOTOMIINEOS (DIPTERA: PSYCHODIDAE) EM FRAGMENTOS

DE FLORESTA AO REDOR DE CONJUNTOS HABITACIONAIS NA CIDADE DE MANAUS,... coletado nesse ambiente foi Psathyromyia que apresentou 4 espécies (3 espécies em cada área). Estes resultados concordaram com diversos estudos realizados neste habitat, na Amazônia central, por Arias \& Freitas (1978), Cabanillas et al. (1995), Cabanillas \& Castellón (1999), Paes (1991) e Barrett et al. (1996).

Diferenças quanto a presença ou ausência de certas espécies de flebotomíneos em troncos de árvores em diversos estudos é notório, mas a nível de subgênero a concordância é unânime. É provável que algumas espécies utilizem este ambiente (troncos de árvores) principalmente como abrigo, como L. umbratilis e $L$. anduzei, mas outras espécies utilizam os troncos de árvores provavelmente como local de repouso alternativo, devido a sua baixa densidade nesses locais e sua maior abundância em outros locais como foliço, tocas de animais, buracos de árvores, entre outros, fato observado nas espécies pertencentes aos subgêneros Psathyromyia e Psychodopygus, além de $L$. (N.) flaviscutellata (Forattini 1973). Fatores como a multiplicidade de abrigos, maior quantidade de animais para repasto sanguíneo, oportunidade de acasalamento e microhabitats estáveis poderiam favorecer para a presença de espécies destes subgêneros em bases de árvores. Mas não podemos deixar de considerar a competição e predação como fatores limitantes e provavelmente a razão para a presença do reduzido número de indivíduos. Estudos visando aspectos acima ressaltados serão de auxílio no esclarecimento da importância destes locais na epidemiologia da LTA na Amazônia Brasileira.

\section{BIBLIOGRAFIA CITADA}

Andrade, S. L.; Fé, N. F.; Fé, F. F.; Toledo, L. M.; Romero, G. A. S. 1999. Leishmaniose tegumentar americana em área de ocupação recente na periferia da cidade de Manaus, estado do Amazonas, Brasil - Avaliação entomológica. Rev. Soc. Bras. Med. Trop.32 (suppl.1): 11-12.

Arias, J. R.; Freitas, R. A. 1982. On the vectors of cutaneous leishmaniasis in central amazon of brasil. 3. Phlebotomine sand fly stratificacion in a terra firme forest. Acta Amazonica, 12 (3): 599-603.

Arias, J. R.; Naiff, R. D. 1981. The principal reservoir host of cutaneous leishmaniasis in urban áreas of Manaus, Central Amazon of Brasil. Mem. Inst. Oswaldo Cruz, 76 (3): 279-286.

Arias, J. R.; Freitas, R. A.; Naiff, R. D.; Barrett, T. V. 1987. Observations on the parasite Leismania mexicana amazonensis and its natural infection of the sand fly Lutzomyia olmeca nociva. PAHO Bulletin, 21 (1): 48-54.

Barrett, T. V.; Senra, M. S. 1989. Leishmaniasis in Manaus, Brazil: Parasitology Today, 5: 255-257.

Barrett, T. V.; Freitas, R. A.; Naiff, M. F.; Naiff, R. D. 1991. A leishmaniose e seus transmissores em relação à saúde na Amazônia. In: Val, A. L.; Figlioulo, R.; Feldberg, E. (Eds.). Bases Científicas para Estratégias de Preservação e Desenvolvimento da Amazônia; Fatos e perspectivas. INPA, Manaus: p.150-117.
Barrett, T. V., Freitas, R. A.; Albuquerque, M. I. C.; Guerrero, J. H. C. 1996. Report on a colecion of Lutzomyia sand fly (Diptera: Psychodidae) from the Middle Solimões (Amazonas, Brasil). Mem. Inst. Oswaldo Cruz. 9 (1): 27-35.

Biancardi, C. B. 1981. Aspectos da epidemiologia da Leishmaniose cutânea na Rodovia BR 364, Território Federal de Rondônia. Dissertação de Mestrado em Entomologia, Instituto Nacional de Pesquisas da Amazônia / Universidade do Amazonas, Manaus, 259pp. il.

Cabanillas, M. R. S.; Castellón, E.G. 1999. Distribution of sandfly (Diptera: Psychodidae) on Tree-trunks in a Non-flooded area of the Ducke Forest Reserve, Manaus, AM, Brazil. Mem. Inst. Oswaldo Cruz, 94 (3): 289-296.

Cabanillas, M. R. S.; Castellón, E.G.B.; Alencar, M. 1995. Estudo sobre os abrigos naturais dos flebotomíneos (Díptera: Psychodidae) na reserva florestal Ducke Manaus, AM, Brasil. Bol. Dir. Malariol. y San. Amb., XXXV, Supl.1: 63-76.

Forattini, O. P. 1973. Entomologia Médica. Ed. Edgard Blucher, São Paulo, Brasil. 658pp.

Gomes, A. C.; Rabello, E. X.; Santos, J. L. F.; Galati, E. A. B. 1982. Aspectos ecológicos da leishmaniose tegumentar americana 2. Ecótopo artificial como abrigo de Psychodopygus intermedius e observações sobre alimentação e reprodução sobre a influência de fatores físicos naturais. Rev. Saúde Publ., 16: 149-159.

Guerra, J.A.O.; Barros, M.L.B.; Guerra, M.V.F.; Talhari, S.; Paes, M.G. 1998. Leishmaniose Tegumentar Americana (LTA) no Município de Manaus - Aspectos epidemiológicos. Rev. Soc. Bras. Med. Trop. 31 (suppl.l): p.172.

Lainson, R. 1983. The American Leishmaniose: some observation on their ecology and epidemiology. Trans. Roy. Soc. Trop. Med. Hyg., 77(5): 569-596.

Lainson, R.; Shaw, J.J.; Souza, A.A.A.; Silveira, F.T.; Falqueto, A. 1992. Futher observations on Lutzomyia ubiquitalis (Psychodidae Phlebotominae), the sandfly vector of Leishmania (Viannia) lainsoni. Mem. Inst. Oswaldo Cruz, 87(3): 437-439.

Marzochi, M.C. A.; Marzochi, K. B. F. 1997. Leishmaniose em áreas urbanas. Rev. Soc. Bras. Med. Trop.,30 (suppl.1): 162-165.

MINISTÉRIO DA SAÚDE - Fundação Nacional de Saúde (MS-FNS) 1994. Guia de controle de leishmaniose tegumentar americana. $3^{\mathrm{a}}$ ed., Brasília, 44pp.

Paes, M. G. 1991. Estudo de quatro espécies de Lutzomyia França, 1924 (Diptera: Psychodidae), em área endêmica de Leishmaniose Tegumentar Americana na periferia de Manaus (Amazonas Brasil). Dissertação de Mestrado. PPG-BTRN - INPA/FUA. 128pp.

Rangel, E. F.; Azevedo, A. C. R.; Lima, J. B.; Souza, N. A.; Pereira, T.; Menezes, C. R. V.; Costa, V.A. 1999. Ecologia da leishmaniose cutânea no estado do Mato Grosso. I. Distribuição Vertical da Fauna flebotomínica (Díptera: Psychodidae; Phlebotominae). Rev. Soc. Bras. Med. Trop., 32 (suppl.1): 25pp.

Ryan, L.; Lainson, R.; Shaw, J. J. 1987. Leishmaniasis in Brazil XXIV. Natural flagellate infections of sandfly (Diptera: Psychodidae) in Pard State, with particular reference to the role of Psychodopygus wellcomei as the vector of Leishmania (Leishmania) brasiliensis in the Serra dos Carajás. Trans.R. Soc. Trop. Med. Hyg., 81: 353-359. 
Teodoro, U.; Salvia-Filho, V. L.; Lima, E. M.; Spinosa, R. P. ; Barbosa, O. C.; Ferreira, M. E. M. C.; Lonardoni, M. V. C. 1993. Observações sobre o comportamento de flebotomíneos em ecótopos florestais e extraflorestais, em área endêmica de leishmaniose tegumentar americana, no norte do Estado do Paraná, sul do Brasil. Rev. Saúde Pública, 27 (4): 287-292.

Tesh, R. 1995. Control of zoonotic visceral leishmaniasis: Is it time change strategies? Am. J. Trop. Med. Hyg., 52 (3): 287-292.

WHO (World Health Organization) / OPS (Organização Panamericana de Saúde) 1996. Leishmaniasis en las Americas. Situacion actual y alternativas para su control. Oficina Sanitária Panamericana, Oficina Regional de la Organización Mundial de Salud, 38pp.
Young, D. G.; Duncan, M.A. 1994. Guide to identification and geographic distribution of Lutzomyia sandfly in México, West Indies, Central and South America (Diptera: Psychodidae). Associated Publishers, American Entomological Institute, 881pp.

RECEBIDO EM 30/11/2002

ACEITO EM 15/07/2003 
